\title{
Role of circadian gene Clock during differentiation of mouse pluripotent stem cells
}

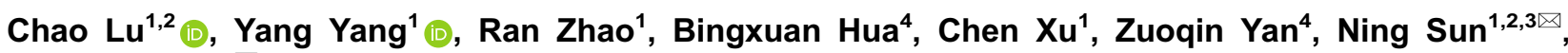 \\ Ruizhe Qian ${ }^{1,2 \bowtie}$ \\ ${ }^{1}$ Department of Physiology and Pathophysiology, School of Basic Medical Sciences, Fudan University, Shanghai 200032, \\ China \\ ${ }^{2}$ Research Center on Aging and Medicine, Fudan University, Shanghai 200032, China \\ 3 State Key Laboratory of Medical Neurobiology, Fudan University, Shanghai 200032, China \\ ${ }^{4}$ Department of Orthopedics, Zhongshan Hospital, Fudan University, Shanghai 200032, China \\ $\triangle$ Correspondence: sunning@fudan.edu.cn (N. Sun), rzqian@shmu.edu.cn (R. Qian) \\ Received May 24, 2016 Accepted August 3, 2016
}

\begin{abstract}
Biological rhythms controlled by the circadian clock are absent in embryonic stem cells (ESCs). However, they start to develop during the differentiation of pluripotent ESCs to downstream cells. Conversely, biological rhythms in adult somatic cells disappear when they are reprogrammed into induced pluripotent stem cells (iPSCs). These studies indicated that the development of biological rhythms in ESCs might be closely associated with the maintenance and differentiation of ESCs. The core circadian gene Clock is essential for regulation of biological rhythms. Its role in the development of biological rhythms of ESCs is totally unknown. Here, we used CRISPR/CAS9-mediated genetic editing techniques, to completely knock out the Clock expression in mouse ESCs. By AP, teratoma formation, quantitative real-time $P C R$ and Immunofluorescent staining, we did not find any difference between Clock knockout mESCs and wild type mESCs in morphology and pluripotent capability under the pluripotent state. In brief, these data indicated Clock did not influence the maintaining of pluripotent state. However, they exhibited decreased proliferation
\end{abstract}

Chao Lu and Yang Yang have contributed equally to this work.

Electronic supplementary material The online version of this article (doi:10.1007/s13238-016-0319-9) contains supplementary material, which is available to authorized users. and increased apoptosis. Furthermore, the biological rhythms failed to develop in Clock knockout mESCs after spontaneous differentiation, which indicated that there was no compensational factor in most peripheral tissues as described in mice models before (DeBruyne et al., 2007b). After spontaneous differentiation, loss of CLOCK protein due to Clock gene silencing induced spontaneous differentiation of mESCs, indicating an exit from the pluripotent state, or its differentiating ability. Our findings indicate that the core circadian gene Clock may be essential during normal mESCs differentiation by regulating $\mathrm{mESCs}$ proliferation, apoptosis and activity.

KEYWORDS Circadian gene Clock, mouse embryonic stem cells, gene knockout, pluripotency, cell proliferation, cell apoptosis, cell differentiation

\section{INTRODUCTION}

Circadian rhythm is a fundamental biological system in humans, animals and plants that regulates various physiological functions such as sleep-wake cycle, energy metabolism, cell division, post-transcriptional regulation (Koike et al., 2012; Cardinal-Aucoin and Steel, 2016; Lopez-Minguez et al., 2016; McAlpine and Swirski, 2016; Nitschke et al., 2016), and endocrine system (Gachon et al., 2004; Lowrey and Takahashi, 2004). The master circadian clock, located in the suprachiasmatic nucleus (SCN), is an essential activator of downstream molecular events critical to 
the generation of circadian rhythms (Dunlap, 1999). The master clock is regulated by four major groups of genes: Clock, Bmal1, Cry (Cryptochrome 1 and 2), and Per (Period 1 and 2). These core circadian genes are regulated in a transcription-translation feedback loop (TTFL) (Xue et al., 2016).

The core circadian genes regulate critical aspects of cellular processes in many organs. Mutations in the circadian genes affect circadian activities and are hazardous to health (King et al., 1997; Kovanen et al., 2010). For instance, some recent studies have shown associations between clock genes and chronic inflammation, blood pressure and energy intake in humans, supporting the importance of the circadian rhythm in cardiac physiology (Dashti et al., 2016; Johnston and Ordovas, 2016). Mutations in Per genes increase cancer incidence (Fu et al., 2002; Wood et al., 2008; Borgs et al., 2009) and cancer cell proliferation (Borgs et al., 2009). Circadian clock dysfunction has been linked to oxidative stress and age-related neurodegeneration in vivo (Witting et al., 1990; Hu et al., 2009; Wyse and Coogan, 2010). Adult stem cell functions are also regulated by circadian oscillations (Casanova-Acebes et al., 2013; Janich et al., 2013; Karpowicz et al., 2013).

Circadian rhythms in the suprachiasmatic nucleus (SCN) manifest only a few days before birth (Yagita et al., 2010). Bmal1 is expressed in mice during embryogenesis without oscillation (Johnson et al., 2002). It may have other key roles instead of circadian rhythm control during embryogenesis (Yang et al., 2016). Indeed, circadian rhythms do not occur in embryonic stem cells (ESCs) but develop when ESCs exit from the pluripotent state and differentiate to downstream cells (Yagita et al., 2010). Conversely, biological rhythms in adult somatic cells disappear when they are reprogrammed into induced pluripotent stem cells (iPSCs). To better understand the molecular basis underlying circadian rhythms and ESCs pluripotency and differentiation, we studied the role of the core circadian gene Clock in the maintenance, differentiation and development of circadian rhythms in mouse embryonic stem cells (mESCs). Using the CRISPR/CAS9 genome editing tool, we completely knocked out the Clock gene in mESCs. We found that Clock knockout mESCs still exhibited normal clonal morphology and pluripotency, which indicated that Clock gene might not be required for regular maintenance of mESCs. However, Clock knockout significantly decreased the proliferation and increased the apoptosis of mESCs. Furthermore, the biological rhythms failed to develop in Clock knockout mESCs after spontaneous differentiation. Loss of CLOCK protein due to Clock gene silencing in mESCs triggered their spontaneous differentiation, leading to stronger expression of genes in the three embryonic germ layers downstream.

Our findings suggest that the core circadian gene Clock influences mESCs differentiation process by regulating mESCs proliferation, apoptosis and activity.

\section{RESULTS}

\section{Knock out of Clock in mESCs using CRISPR/CAS9} genomic editing

To investigate the role of the core circadian gene Clock in the maintenance and differentiation of mammalian pluripotent stem cells, we established the Clock knockout mESC line using the CRISPR/CAS9 genomic editing tool. This tool enables the efficient construction of knockout alleles via induction of frameshift mutations. In this study, we designed a single gRNA specifically targeting exon 2 in the mouse Clock gene (Fig. 1A) and subcloned it into the PX330 CRISPR/CAS9 vectors. Single wild type mESCs were transfected with the subcloned CRISPR/CAS9 plasmids via electroporation. Single colonies with successful genome editing were selected by puromycin and Sanger's sequencing at the cutting site on exon 2 of the Clock gene (Fig. 1B and Fig. S1). We deleted 31 single mESC clones, of which 8 clones (clone 5, clone 13, clone 17, clone 19, clone 20 , clone 23 , clone 24 , and clone 28 ) showed sequence modifications at the CRISPR/CAS9 cutting site (Table S2). To verify whether the Clock gene was completely knocked out, we investigated CLOCK protein expression in the 8 positive clones using Western blot. The results indicated that CLOCK protein expression was totally ablated in 3 clones (clone 17, clone 19, and clone 20) (Fig. 1C). To further confirm whether both alleles of the Clock gene were modified in clone 17, clone 19 , and clone 20, we performed TA cloning of the PCR products flanking the CRISPR/CAS9 cutting site. The results showed that both alleles were edited at exon 2 of the Clock gene in all the 3 homozygous mESC clones (Fig. 1D and Fig. S1). Among the 3 clones, clone 19 was used for further study.

Further, to consider the potential influence of off-target gene modifications (Yee, 2016), we detected the most probable ten off-target modification sites which were designed at the CRISPR website (http://crispr.mit.edu/) (Table S3) and found that there was no off-target gene modification (Fig. S2), which excluded the potential influence of off-target gene modifications.

\section{Clock knockout in mESCs did not affect pluripotency}

The Clock knockout mESCs exhibited compact clonal morphology without spontaneous differentiation under routine mESC maintenance culture conditions, which was similar to the wild type mESCs (Fig. 2A). To further examine whether silencing of Clock gene affected the pluripotency of mESCs, we performed staining for alkaline phosphatase and immunofluorescence of pluripotent markers OCT4, NANOG, and SSEA1. The Clock knockout mESCs expressed strong alkaline phosphatase activity as well as OCT4, NANOG, and SSEA1 (Fig. 2B and Fig. 3). Real-time PCR of the mRNA expression levels of pluripotent markers Oct4, Sox2, Nanog, KIf4, Zfp296, Dax1, Esg1, and Eras revealed no significant 
A

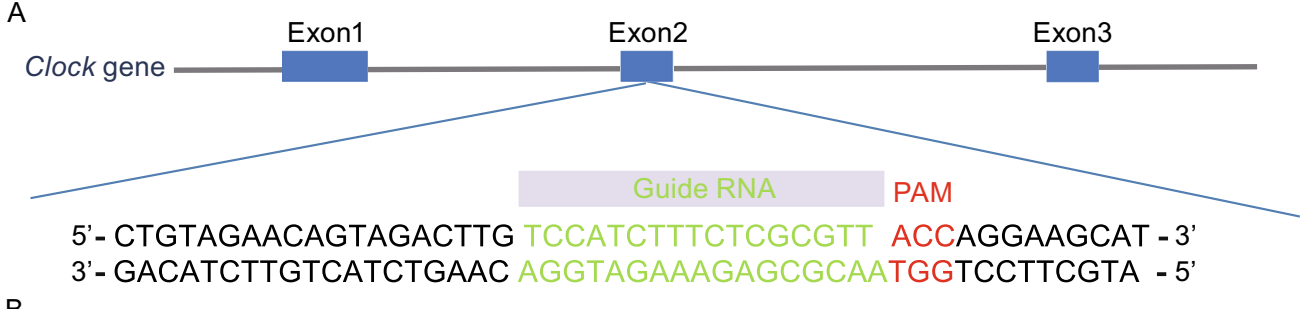

$\mathrm{B}$

WT
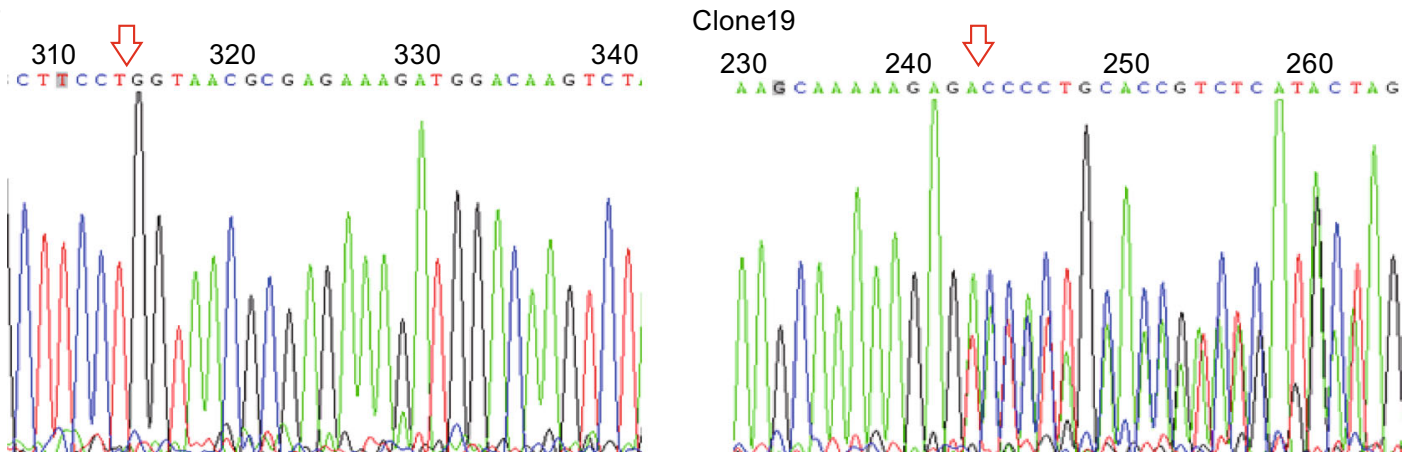

C
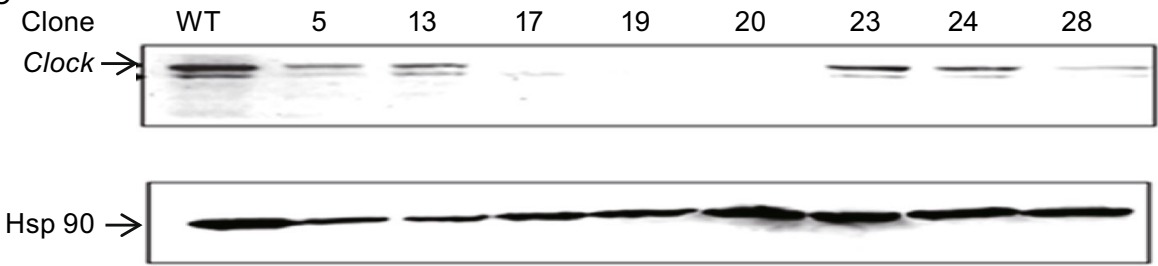

D

Clone 19

$1^{\text {st }}$ Allele

WT: ATGCTTCCTGGT AACGCGAGAAA GATGGACAAGTCTACTGTTCTACAG

Clone19: ATGCTTCCTGGT - GATGGACAAGTCTACTGTTCTACAG

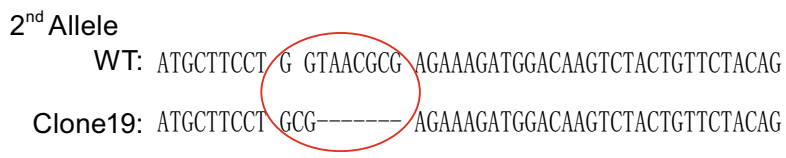

Figure 1. Generation of Clock knockout mESC line using CRISPR/CAS9 gene editing. (A) Schematic diagram of the $5^{\prime}$ end of the Clock gene (blue bars, exons), the CRISPR/CAS9 gRNA sequence (red bar, the green shaded part), PAM domain (the red shaded part). (B) Representative sequencing showing modifications at the CRISPR/CAS9 cutting site on exon 2 of the mouse Clock gene. (C) Western blot revealed CLOCK expression in the positive clones following editing by the CRISPR/CAS9 system. (D) TA cloning results involving a representative homozygous clone (clone 19) show that both alleles of the mouse Clock gene were modified on exon 2 by the CRISPR/Cas9 system.

differences $(P>0.05)$ from those of wild type mESCs (Fig. 2C). Furthermore, we used teratoma formation assay as a tool for monitoring pluripotency in stem cell research to study the pluripotency of wild type mESCs and Clock knockout mESCs in vivo (Fig. 2D) (Nelakanti et al., 2015). As shown in Fig. 2D, after injecting $1 \times 10^{7}$ wild type mESCs or Clock knockout mESCs into immunodeficiency mice, we could observe teratoma of 1-2 cm formed after four weeks. Six weeks later, we detected the differential capacity of wild type mESCs and Clock knockout mESCs by hematoxylineosin staining (HE staining). We found that that both wild type mESCs and Clock knockout mESCs could differentiate to endoderm, mesoderm, and ectoderm layers cells, which indicated that wild type mESCs and Clock knockout mESCs have similar potential of multilineage differentiation in vivo. According to these results we got, silencing of Clock gene in mESCs didn't affect their pluripotent nature.

\section{Clock silencing in mESCs decreased cell proliferation rate}

During routine culture, we observed that the proliferation rate of Clock knockout mESCs was much slower than that of the wild type mESCs. To further confirm our observation, we 
A
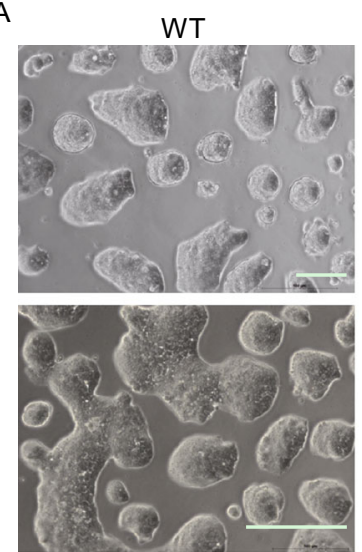

B

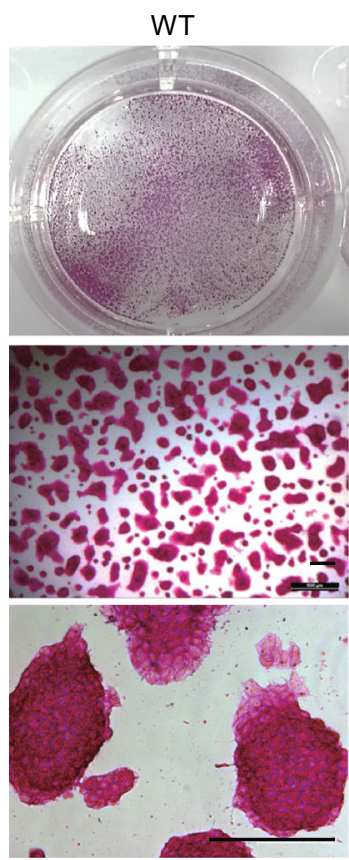

Clock-/-
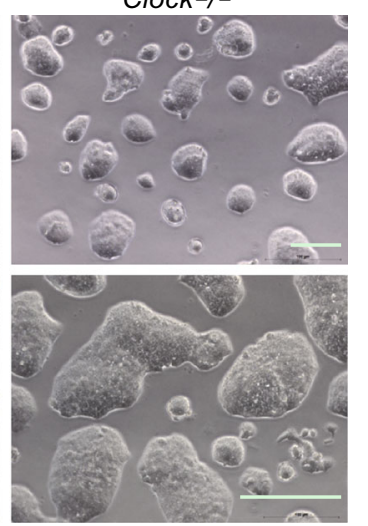

Clock-/-
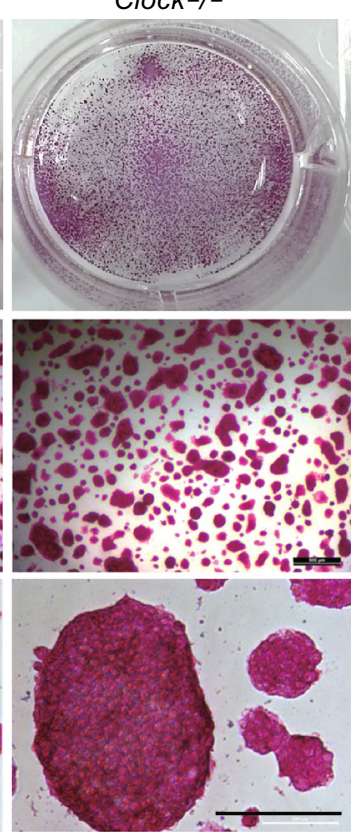

C

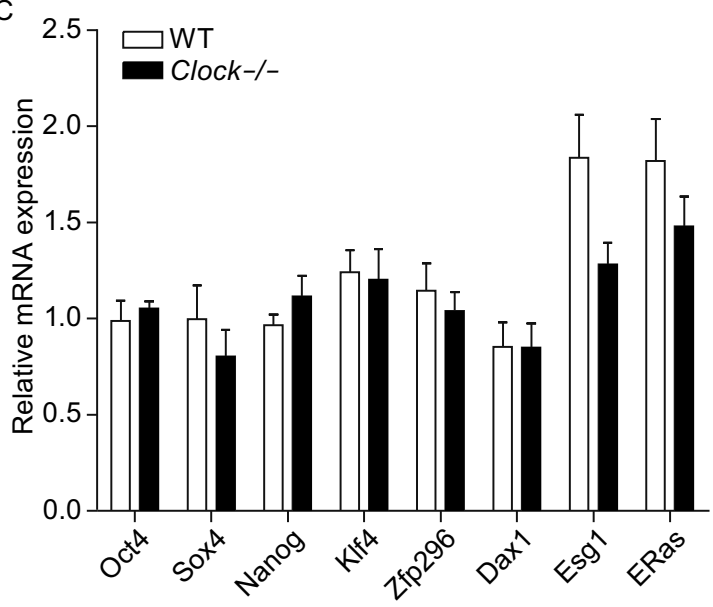

D
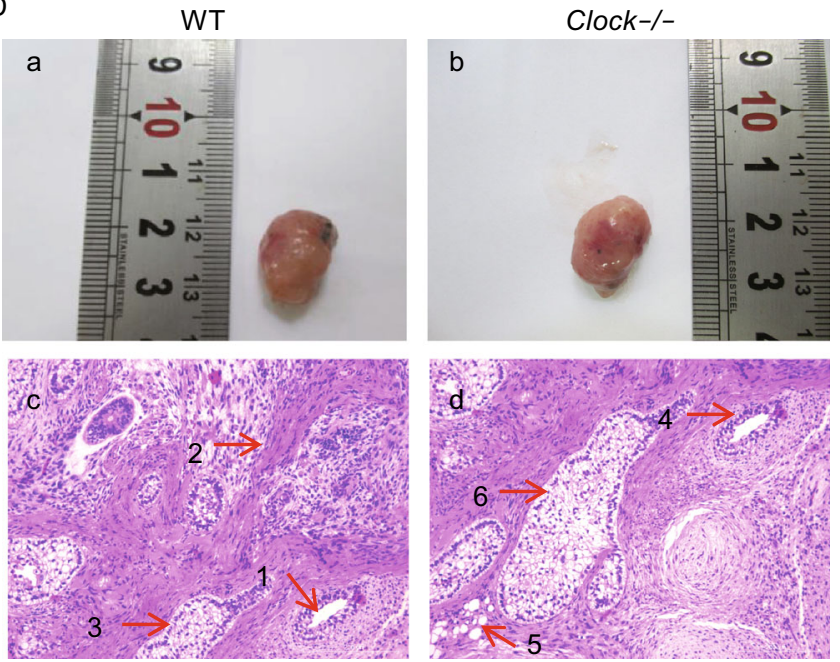

Figure 2. Knockout of Clock did not affect pluripotency of mESCs. (A) Phase images of wild type and Clock-/- mESCs exhibited compact and round clonal morphology. Bars, $200 \mu \mathrm{m}$. (B) Both wild type and Clock-/- mESCs stained strongly for alkaline phosphatase. The upper two images represent macroscopic views of a 12-well plate system. Bars, $400 \mu \mathrm{m}$. (C) Relative mRNA expression of pluripotent markers Oct4, Sox2, Nanog, Klf4, Zfp296, Dax1, Esg1, and Eras in wild type and Clock-I- mESCs. The mRNA expression levels were normalized to that of the endogenous GAPDH. Data represent an average of three independent experiments and are expressed as means \pm S.D. Statistical significance was calculated using the Student's $t$-test: * denotes $P<0.05$, ** denotes $P<0.01$, ${ }^{* * *}$ denotes $P<0.001$ compared with the Ctrl group, ns, not significant. No statistical significance was found in the expression levels of the above genes between the wild type and Clock-/- mESCs $(P>0.05)$. (D) After respectively injecting $10^{7}$ wild type mESCs and Clock knockout mESCs into immunodeficiency mice, we could observe teratoma of similar size (1-2 cm) formed after four weeks. Six weeks later, Immunohistochemistry manifested that both wild type mESCs and Clock knockout mESCs could differentiate to downstream endoderm, mesoderm, and ectoderm layers cells. Among them, arrow 1 and 4 denote gland cells, which belong to endoderm layer cells; arrow 2 denotes smooth muscle cells, and arrow 5 denotes fat vesicle cells, which belong to mesoderm layer cells; arrow 3 and 6 denote sebaceous gland cells, which belong to ectoderm layer cells.

performed cell proliferation assays of the Clock knockout mESCs and wild type mESCs at different time points. As shown in Fig. 4A, the Clock knockout slowed the proliferation rate of mESCs significantly compared with that of the wild type mESCs $(P<0.001)$. These results indicated that Clock knockout negatively affected the proliferation of mESCs. 
A
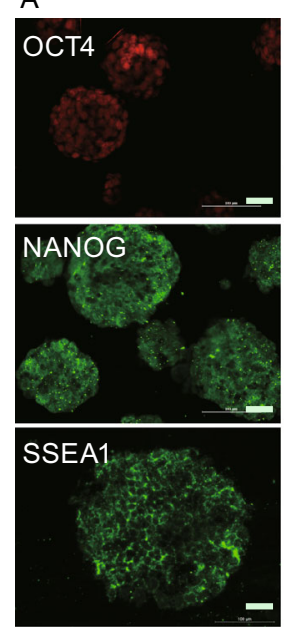

B
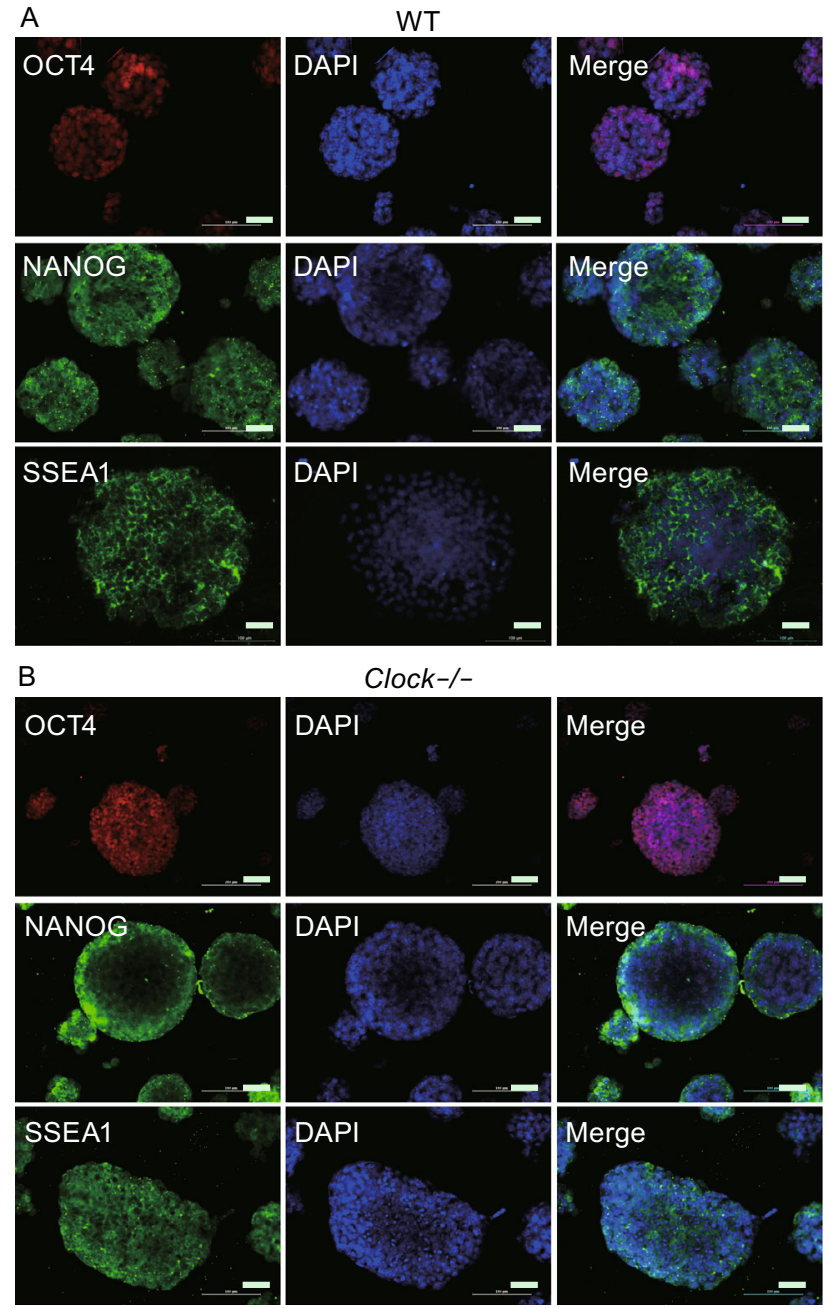

Clock-/-
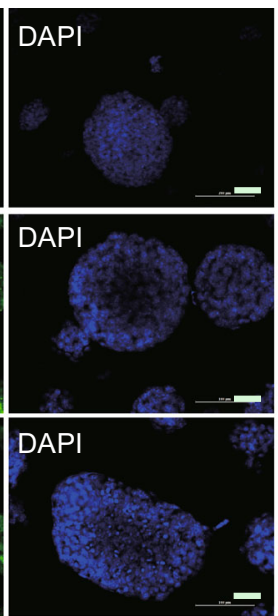
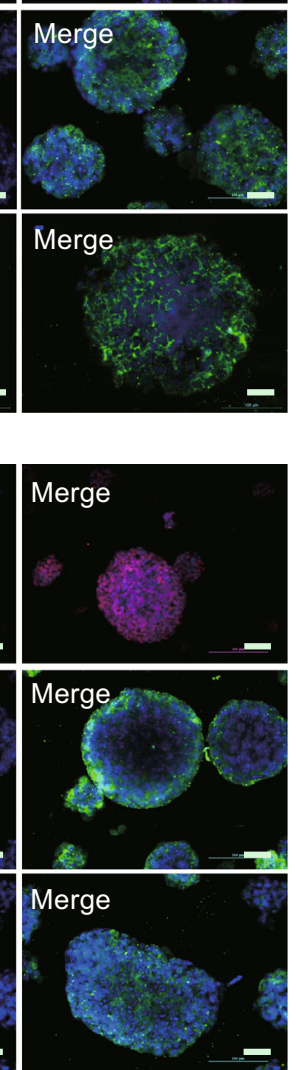

Figure 3. Clock-/- mESCs express pluripotent markers OCT4, NANOG, and SSEA1. (A) Immunofluorescence staining of the pluripotent markers OCT4, NANOG, and SSEA1 in wild type mESCs. (B) Immunofluorescence staining of the pluripotency marker OCT4, NANOG, and SSEA1 in Clock-/- mESCs. Bars, $100 \mu \mathrm{m}$.

Clock knockout in mESCs slowed down the cell cycle and enhanced cell death

The decreased proliferation of the Clock knockout mESCs suggested either a slowdown of cell cycle or increased cell death in mESCs. To test our hypotheses, we first examined the expression of cell cycle-related markers C-Myc, CyclinD1, CDK1, CDK2, PCNA, and $P 27$ by real-time PCR in the Clock knockout mESCs and compared with those in the wild type mESCs. We found that the expression levels of C-Myc, CyclinD1, CDK1, CDK2, and PCNA, which facilitated cell proliferation, were lower in Clock knockout mESCs than in the wild type mESCs $(P<0.05, P<0.01)$. The P27 mRNA expression, which restrained cell proliferation, was higher in Clock knockout mESCs than in the wild type mESCs (Fig. 4B). These results indicated that Clock silencing in

mESCs decreased the cell cycle. To determine whether the decreased proliferation also resulted from increased cell death, we further investigated cell death in the Clock knockout mESCs and the wild type mESCs. Based on six independent experiments using FACS analysis of apoptosis with Annexin V and PI (Propidium lodide), respectively, indicated that the Clock knockout mESCs exhibited significantly higher rate of apoptosis and cell death compared with the wild type mESCs $(P<0.05$ and $P<0.01)$ (Fig. 5A and B). To further verify this result, we determined the mRNA expression levels of apoptosis-related markers Bax, Bcl-2, caspase 3, and caspase 9 in the Clock knockout mESCs and the wild type mESCs. As shown in Fig. 5C, the mRNA expression levels of Bax, caspase 3, and caspase 9, which were associated with enhanced apoptosis, were higher in the Clock knockout mESCs than in the wild type mESCs $(P<$ 0.05). The expression level of $B c l-2$, which was associated with decreased cell apoptosis, was lower than in the wild type mESCs $(P<0.05)$. These results demonstrated that silencing of Clock increased cellular apoptosis, resulting in increased cell death of mESCs.

\section{Clock is required for development of circadian} oscillator during mESCs differentiation

Pluripotent mESCs do not exhibit molecular circadian oscillations. However, circadian rhythms developed during the differentiation of pluripotent mESCs into downstream lineages (Yagita et al., 2010). Whether silencing of the Clock gene altered the development of circadian oscillation of mESCs during differentiation remains unknown. Therefore, we analyzed the expression of the core clock genes Bmal1, Clock, Per, and Cry on day 15 after spontaneous differentiation of the Clock knockout mESCs and wild type mESCs. Consistent with a previous report, the wild type mESCs developed circadian oscillations on day 15 following spontaneous differentiation. In contrast, the Clock knockout mESCs exhibited no circadian oscillation on day 15 after spontaneous differentiation (Fig. 6). These results indicated that silencing of the gene Clock disrupted the development of circadian oscillations during the differentiation of pluripotent mESCs.

\section{Clock knockout in mESCs accelerated spontaneous differentiation}

We next investigated whether Clock knockout and disruption of the normal circadian oscillations affected mESCs differentiation. $1 \times 10^{4}$ of wild type mESCs or Clock knockout $\mathrm{mESCs}$ were cultured in differentiation medium. Surprisingly, after day 7, Clock knockout mESCs showed more complex cellular structures as well as faster proliferation rate after spontaneous differentiation (Fig. S3A). The mRNA expression levels of the pluripotent markers Oct4, Sox2, Nanog, and KIf4 were gradually decreased in both the Clock 

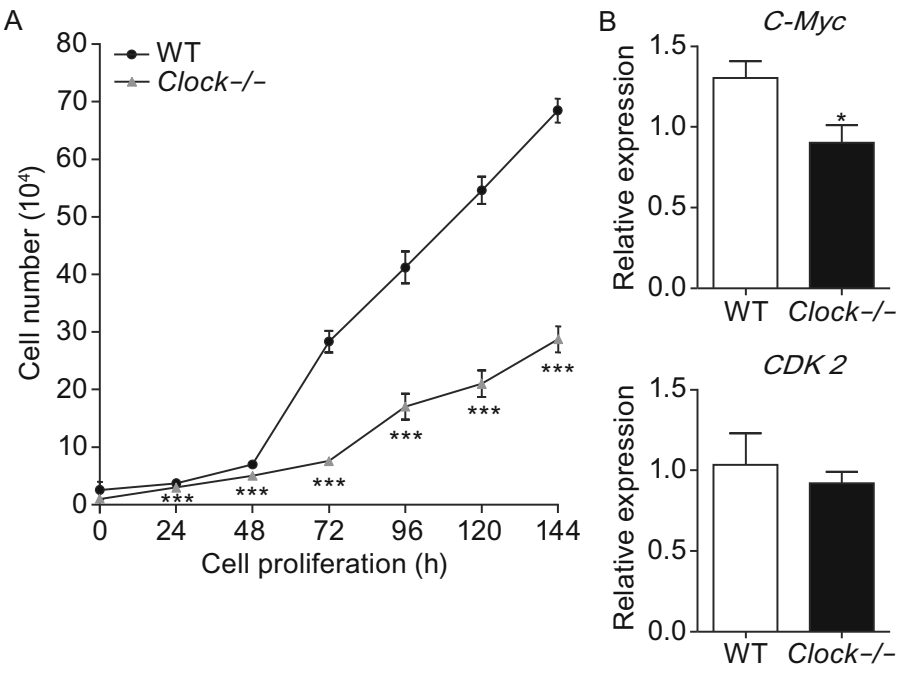

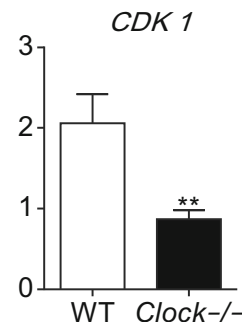

PCNA

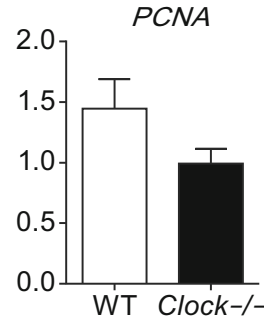

CyclinD 1

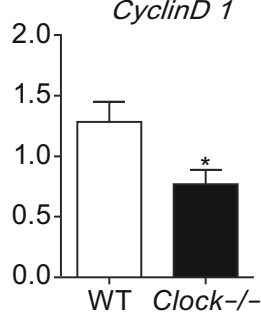

$P 27$

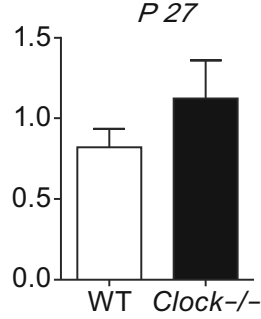

Figure 4. Clock silencing inhibited proliferation of mESCs. (A) Cell proliferation curve of the wild type and Clock-/- mESCs. Clock-/- mESCs exhibited significantly lower proliferation rate than that of the wild type mESCs. (B) Relative mRNA expression levels of cell cycle-related markers C-Myc, CyclinD1, CDK1, CDK2, PCNA, and P27 in wild type and Clock-/- mESCs. The mRNA expression levels were normalized to that of the endogenous $G A P D H$. Data represent an average of three independent experiments and are expressed as means \pm S.D. Statistical significance was calculated using the Student's $t$-test: * $\operatorname{denotes} P<0.05$, ${ }^{* *}$ denotes $P<0.01,{ }^{* * *}$ denotes $P<0.001$ compared with the Ctrl group, ns, not significant.

knockout mESCs and wild type mESCs after spontaneous differentiation (Fig. 7A), indicating an exit from the pluripotent state. Spontaneous differentiation also led to a gradual increase in the mRNA expression levels of the endoderm markers Gata4, Sox17, Foxa2, and Lamb1 (Fig. 7B), the mesoderm markers BMP4, T, Eomes, and Actc1 (Fig. 7C), as well as the ectoderm markers Nestin, Sox1, Neurod1, and Otx1 (Fig. 7D). Interestingly, the mRNA expression levels of the above markers in the three embryonic germ layers (ectoderm, mesoderm, and endoderm) in the Clock knockout mESCs were relatively higher than in the wild type mESCs. The Clock knockout mESCs showed accelerated differentiation compared with wild type mESCs. To further verify these observations, we used immunofluorescence staining of the endoderm marker FOXA2 and the ectoderm marker SOX1 in both the Clock knockout mESCs and wild type mESCs on day 9 after spontaneous differentiation. The FOXA2 and SOX1 stained strongly in the Clock knockout mESCs than in the wild type mESCs (Fig. 8A and B). These results suggested that knockout the Clock triggered spontaneous differentiation of mESCs.

\section{DISCUSSION}

In this study, we investigated the role of the core circadian gene Clock in the maintenance and differentiation of pluripotent stem cells. We used the CRISPR/CAS9 genome editing tool to induce frameshift mutations in exon 2 of the mouse Clock gene and completely ablated its protein expression. This is the first report of a complete knockout of Clock expression in pluripotent stem cells. Previous studies mostly used siRNA/shRNA-mediated silencing of Clock expression (Mukherjee et al., 2010; Tobback et al., 2012; Tracey et al., 2012; Liang et al., 2013; Li et al., 2015). The complete knockout of Clock in our study avoided any potential noise observed in partial knockdown studies involving siRNA/shRNA.

The knockout of the gene Clock did not disrupt the clonal morphology or pluripotency of mESCs. However, loss of Clock significantly decreased the proliferation and increased the cell death of mESCs. Our findings are consistent with other studies that reported a potential relationship between Clock gene and apoptosis as well as cell cycle regulation. One study demonstrated that upregulation of several proapoptic genes in the spleen of Clock mutant mouse (Gaddameedhi et al., 2015). Another study also reported that the Clock gene controlled the expression of key cell cycle-related regulators, such as Cdc2, Wee1, P21, PCNA and Cdk2 in the intestine (Peyric et al., 2013). Our data also showed that the expression of cell cycle-related proteins in Clock knockout mESCs, was affected. The expression of antiapoptotic protein $\mathrm{Bcl}-2$ significantly decreased in Clock knockout mESCs. The expression of pro-apoptotic protein Bax, cleaved caspase-3, and caspase- 9 significantly increased in Clock knockout mESCs. These data suggested a disruption in the balance of mitochondria and activation of the caspase cascade in Clock knockout mESCs (Liang et al., 2013). Clock may, thus, contribute to the maintenance of 


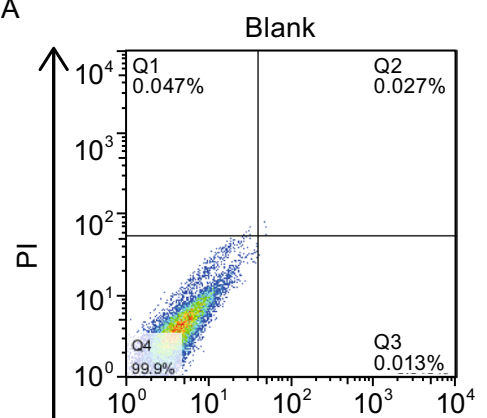

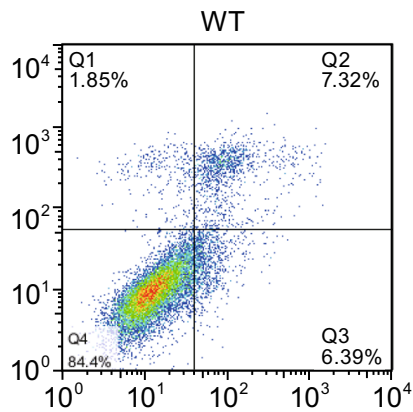
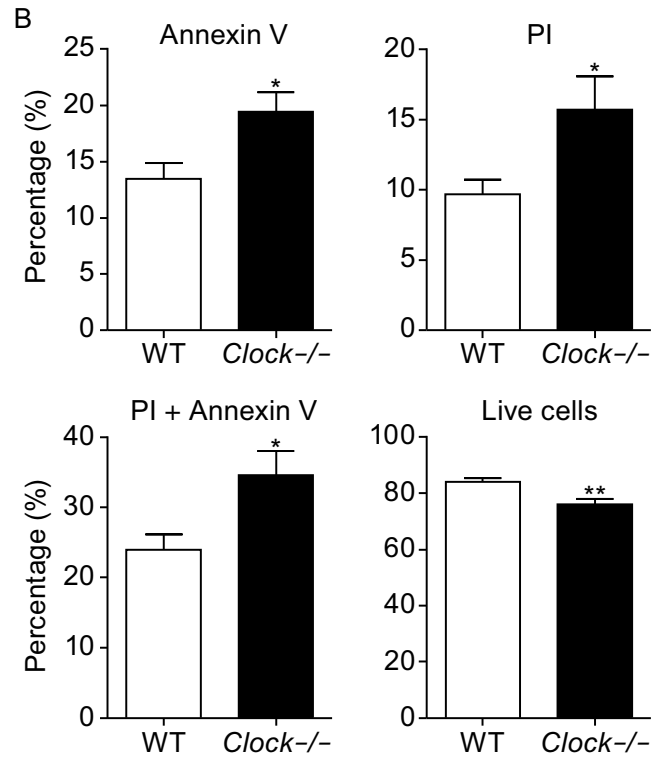

$\bar{\alpha}$

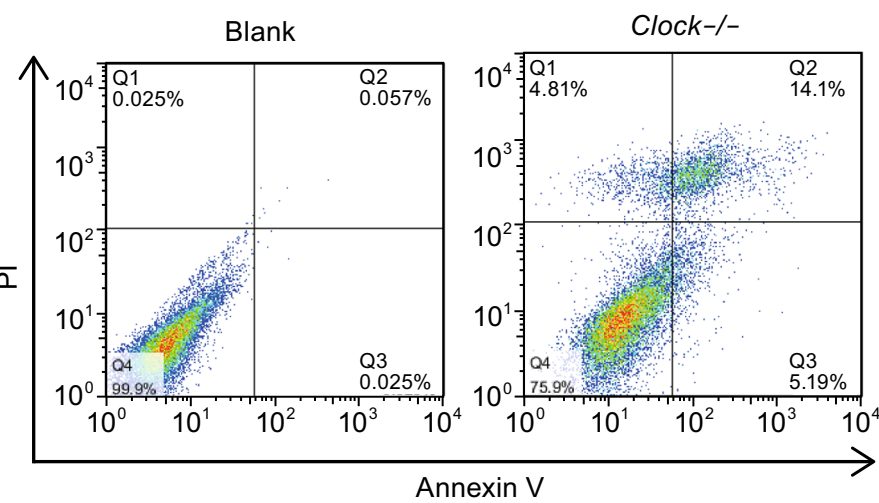

$$
\text { C }
$$

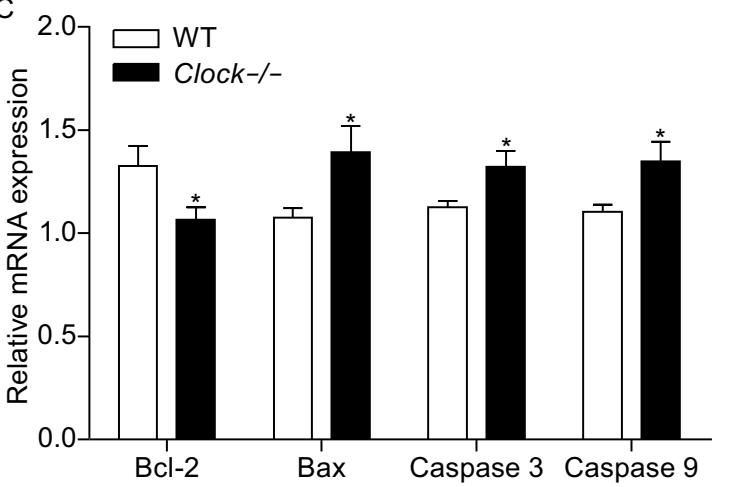

Figure 5. Clock silencing accelerated cell death in mESCs. (A) Apoptosis and overall cell death of the wild type and Clock-/mESCs were analyzed by flow cytometry using the Annexin V and PI assay, respectively. Clock-/- mESCs exhibited increased apoptosis and overall cell death. (B) Percentage of apoptotic (Annexin V) and dead (PI) cells calculated from six independent experiments. Data were expressed as means \pm S.D. (C) Relative mRNA expression levels of apoptosis-related genes Bax, Bcl-2, cleaved caspase-3, and caspase- 9 in wild type and Clock-/- mESCs. The mRNA expression levels were normalized to that of the endogenous $G A P D H$. Data represent the average of three independent experiments and are expressed as means \pm S.D. Statistical significance was calculated using the Student's $t$-test: * denotes $P<0.05$, ${ }^{* *}$ denotes $P<0.01$, ${ }^{\star * *}$ denotes $P<0.001$ compared with the Ctrl group, ns, not significant.

normal proliferation by controlling the balance of cell cycle and apoptosis in mESCs. Overall, our results provide some new insights into the function of the Clock gene in the regulation of cell cycle and apoptosis of mESCs.

To consider the potential influence of off-target gene modifications (Yee, 2016), we detected the most probable ten offtarget modification sites which were designed at the CRISPR website (http://crispr.mit.edu/) (Supplemental Table. 3) and found that there was no off-target gene modification (Supplemental Fig. 2), which excluded the potential influence of offtarget gene modifications. And we further considered the potential influence of alternative cellular roles for Clock such as the transcription factor neuronal PAS domain protein 2
NPAS2 (MOP4), which was able to functionally substitute for the loss of Clock in the SCN but not in peripheral tissues in mice to regulate circadian rhythmicity (DeBruyne et al., 2007a, b; Debruyne, 2008). In our study, we tested the mRNA expression level of NPAS2 in the wild type mESCs and Clock knockout mESCs after spontaneous differentiation, and we found that both of them barely expressed NPAS2 after spontaneous differentiation. This indicated that the nerve cells was very few in wild type mESCs and Clock knockout mESCs after spontaneous differentiation, so there wasn't compensation of NPAS2 for the loss of Clock (Fig. S3B). All in all, these data indicated that the phenotypes in these Clock knockout mESCs should mainly be due to the loss of Clock but not the influence 


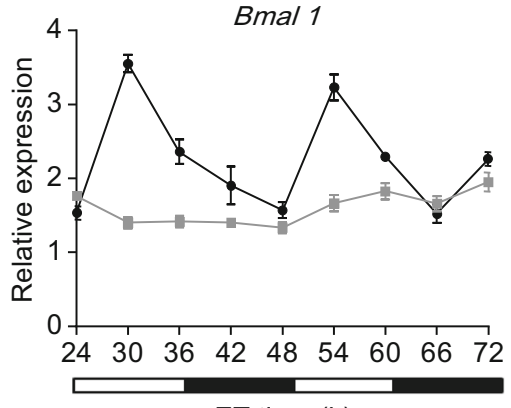

ZT time (h)

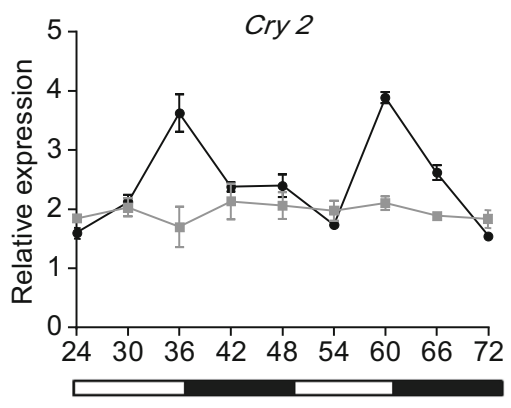

ZT time (h)

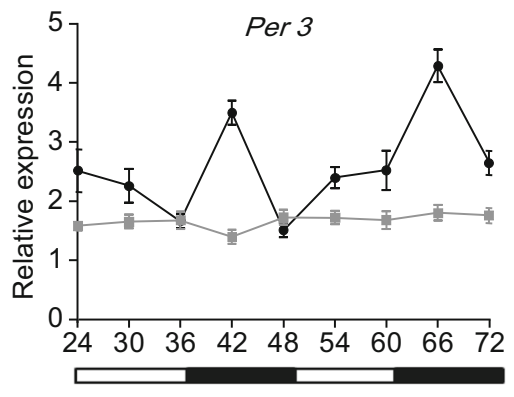

ZT time (h)

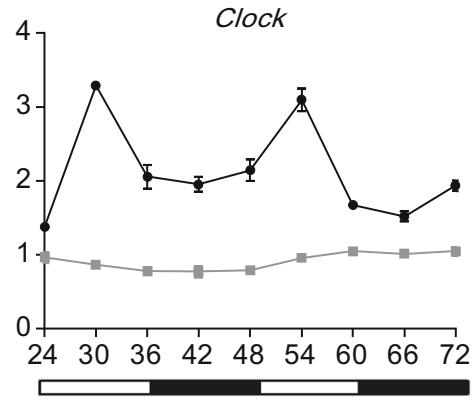

ZT time (h)

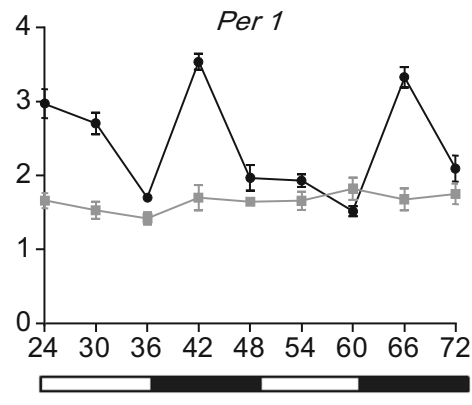

ZT time (h)

$\rightarrow$ WT

- Clock-/-

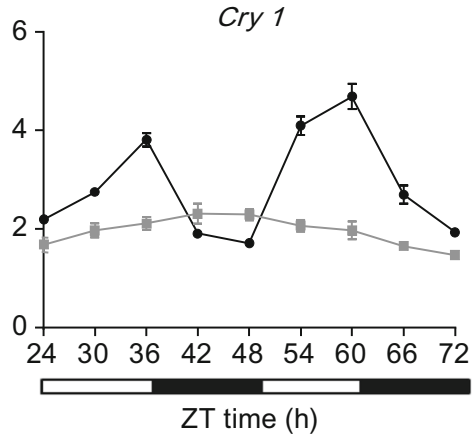

Per 2

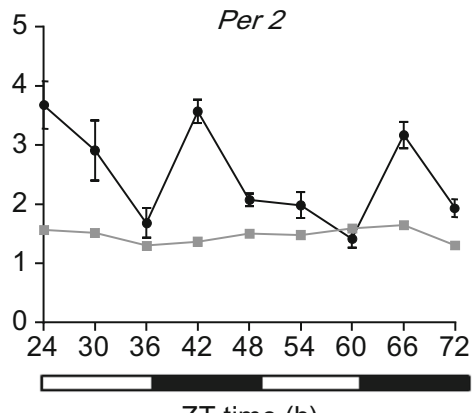

ZT time $(\mathrm{h})$

Figure 6. Clock silencing disturbed the circadian oscillations during mESC differentiation. Relative mRNA expression of core clock genes Bmal1, Clock, Cry1, Cry2, Per1, Per2, and Per3 in spontaneously differentiated wild type and Clock-/- mESCs after serum shock induced biological rhythms. ZT0 to 72 represent 8:00 am to 8:00 am of day 4, respectively. The mRNA expression levels were normalized to that of the endogenous GAPDH. Compared with wild type mESCs, Clock-/- mESCs showed no circadian oscillation in the expression of core clock genes. Data represent the average of three independent experiments and are presented as means \pm S.D.

of off-target gene modifications or loss of other Clock functions.

A recent study has shown that the mammalian circadian oscillator in ground state naïve pluripotent stem cells was not developed until after differentiation (Yagita et al., 2010). We also found in this study that naive mESCs did not exhibit circadian oscillations until differentiation, while Clock was required for the development of this circadian oscillator in mESCs after differentiation. This finding suggested that Clock mediated developmental functions in mammals. Indeed, we found that loss of Clock in mESCs triggered spontaneous differentiation. Several critical genes regulating development of the three emryonic germ layers were expressed higher in the Clock knockout mESCs, which exhibited accelerated spontaneous differentiation in vitro. The disruption of biological rhythms underlying differentiation of Clock knockout mESCs may drive these cells toward spontaneous differentiation upon withdrawal of pluripotent signals. Our study indicated that Clock medicated the differentiation of mouse pluripotent stem cells. Loss of Clock significantly decreased the proliferation and increased the cell death of mESCs indicated that Clock accurately regulated the development of mESCs. Clock knockout in mESCs accelerated spontaneous differentiation. Some studies have reported that Clock knockout in mice is associated with aging and chronic inflammation (Dubrovsky et al., 2010), indicating that this acceleration might be harmful. Our findings indicated that Clock knockout mESCs can conduct one 

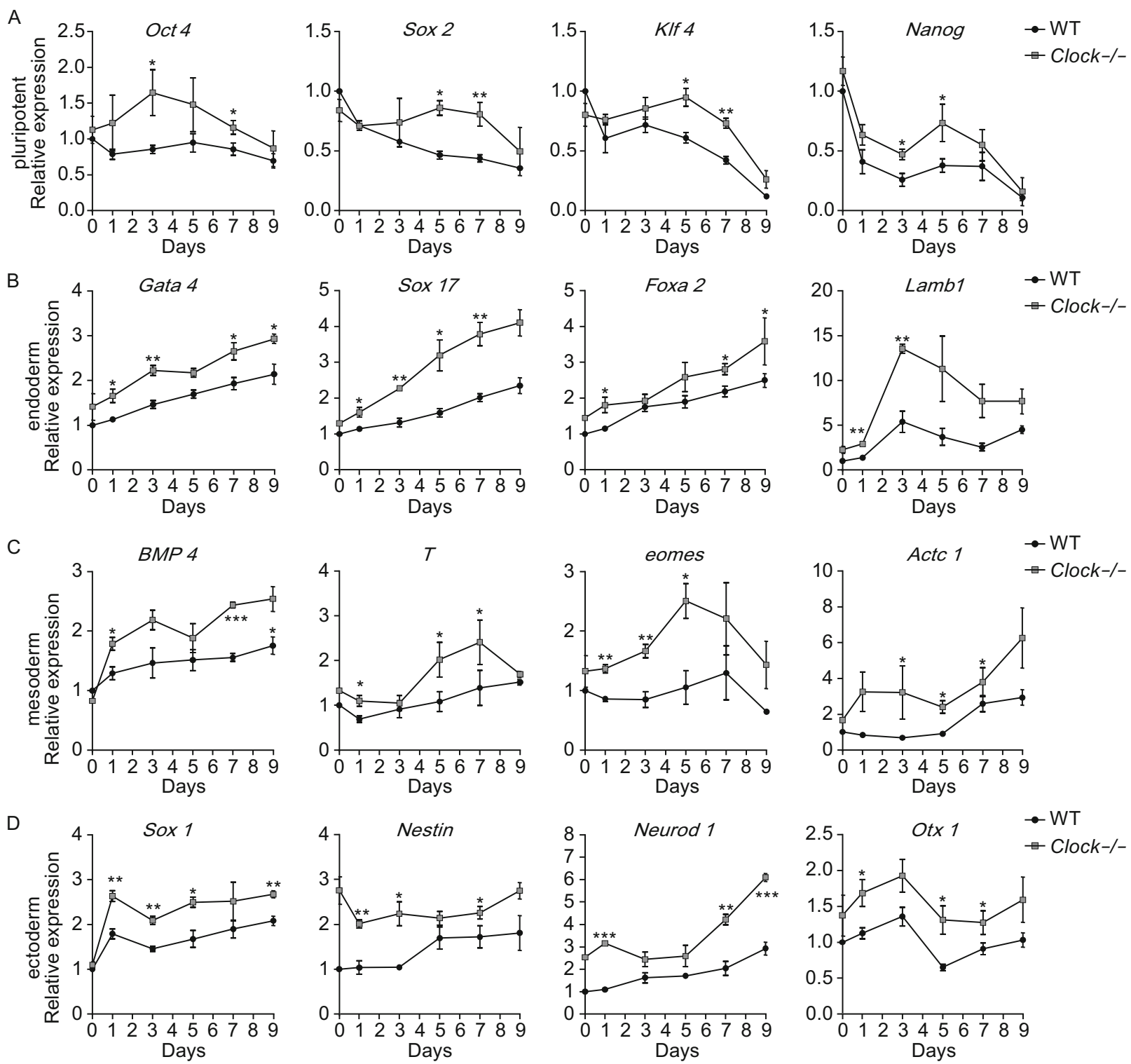

Figure 7. Clock silencing promoted the expression of genes controlling the three embryonic germ layers in spontaneously differentiated mESCs. (A) Relative mRNA expression of pluripotent markers Oct4, Sox2, Nanog, and KIf4 after spontaneous differentiation in wild type and Clock-/- mESCs. Expression of the pluripotent genes was gradually decreased in wild type and Clock-/mESCs. (B) Relative mRNA expression of endoderm markers Gata4, Sox17, Foxa2, and Lamb1 after spontaneous differentiation in wild type and Clock-/- mESCs. Clock-/- mESCs exhibited a higher expression of these endoderm genes. (C) Relative mRNA expression of mesoderm markers BMP4, T, Eomes, and Actc1 after spontaneous differentiation in wild type and Clock-/- mESCs. Clock-/- mESCs exhibited a higher expression of mesoderm genes. (D) Relative mRNA expression of ectoderm markers Nestin, Sox1, Neurod1, and Otx1 after spontaneous differentiation in wild type and Clock-/- mESCs. Clock-/- mESCs exhibited a higher expression of these ectoderm genes. The mRNA expression levels were normalized with that of the endogenous GAPDH. Data represent the average of three independent experiments and are presented as means \pm S.D. Statistical significance was calculated using the Student's $t$-test: ${ }^{*}$ denotes $P<0.05,{ }^{* *}$ denotes $P<0.01,{ }^{* *}$ denotes $P<0.001$ compared with the Ctrl group, ns, not significant.

tool to study these diseases. Yet, additional studies are required to uncover the roles of Clock in mammalian development.
In summary, we found that the core circadian gene Clock was dispensable for maintaining pluripotency in mouse pluripotent stem cells. However, it was required for 


\section{A}
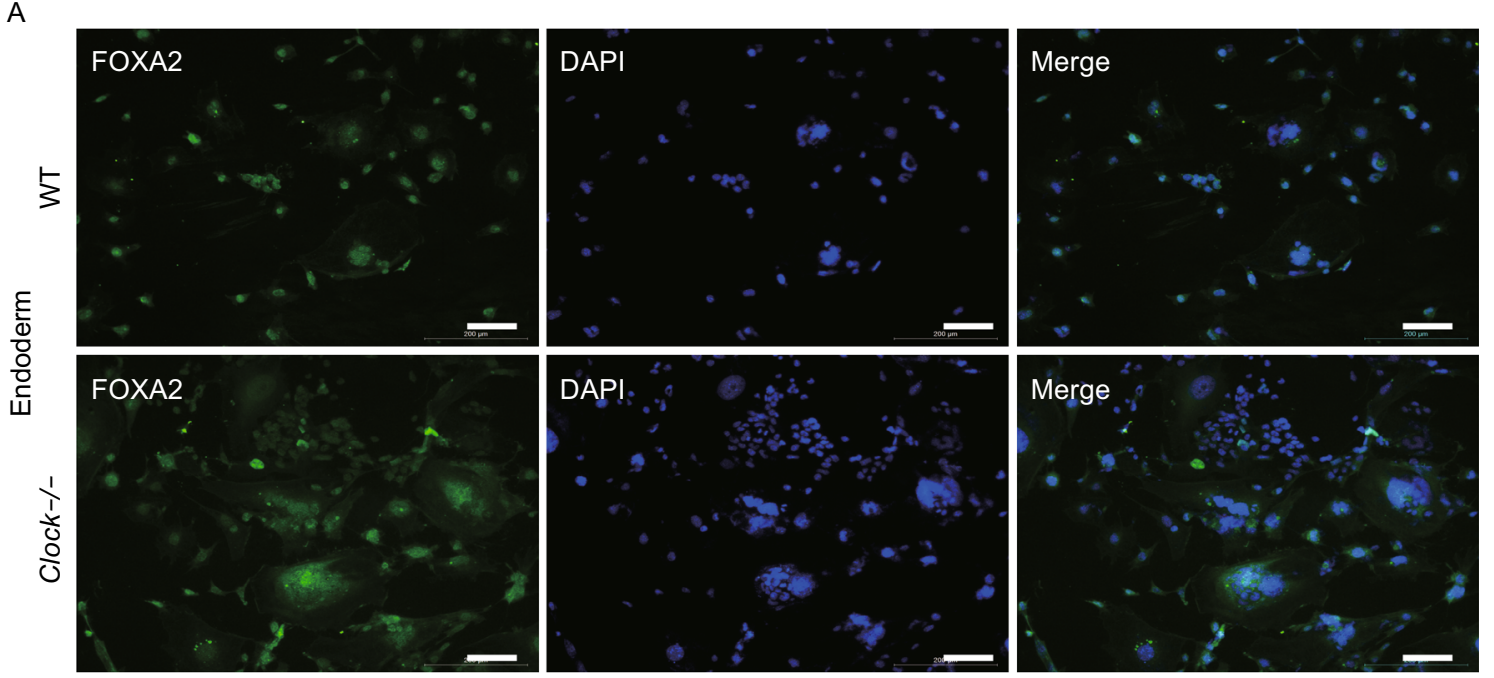

B
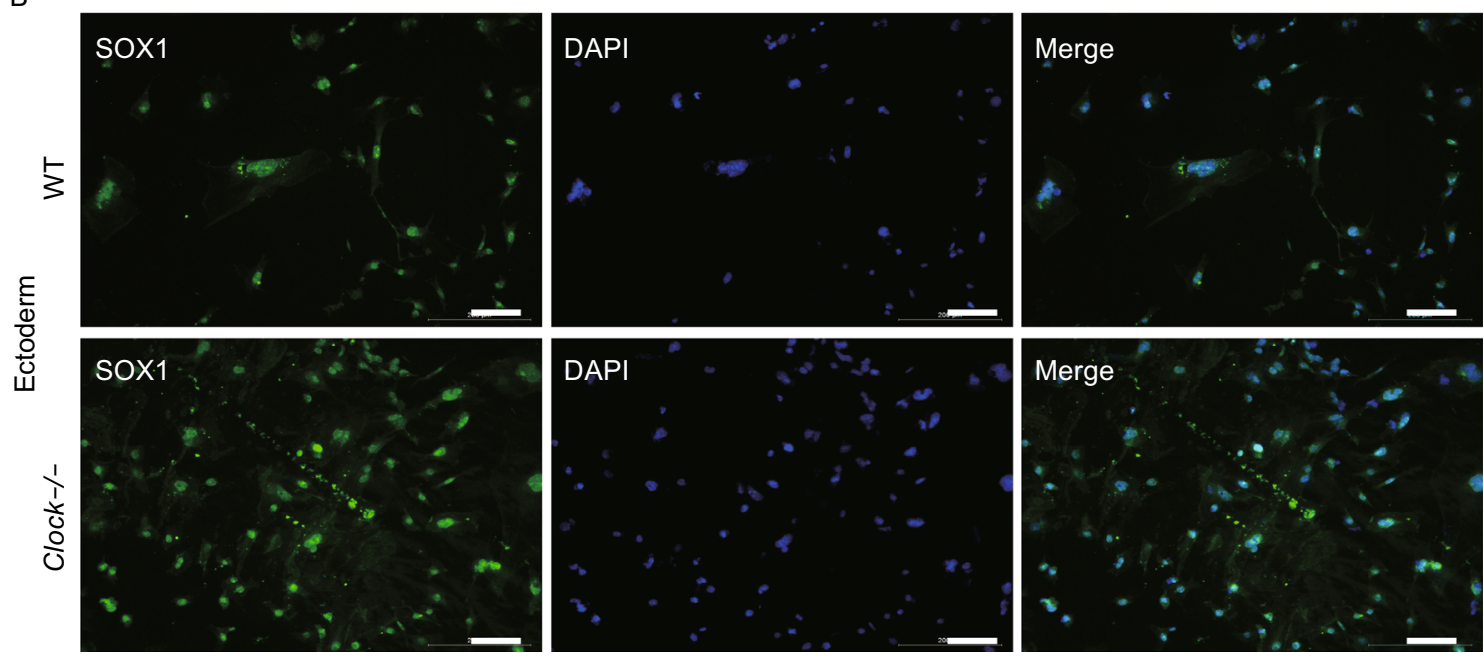

Figure 8. Clock-/- mESCs expressed higher endoderm marker FOXA2 and ectoderm marker SOX1 on day 9 after spontaneous differentiation. (A and B) Immunofluorescence staining of the endoderm marker FOXA2 and the ectoderm marker SOX1 in spontaneously differentiated wild type and Clock-/- mESCs on day 9. Clock-/- mESCs exhibited relatively stronger expression of both FOXA2 and SOX1. Bars, $200 \mu \mathrm{m}$.

maintaining regular proliferation and cell death in mESCs. And Clock was indispensable for the development of circadian oscillations after differentiation of pluripotent stem cells. Furthermore, our data indicated that Clock was essential for the differentiation of mouse pluripotent stem cells. Our findings may provide some new insights into the regulatory mechanisms of Clock in pluripotent stem cell development in mammals.

\section{MATERIALS AND METHODS}

\section{Culture of mESCs}

Mouse 129 ESCs were obtained from ATCC (American Type Culture Collection, Manassas, VA). The wild type and Clock knockout
mESCs were cultured in the maintenance medium of Dulbecco's Modified Eagle's Media (DMEM, Gibco, USA) supplemented with $3.7 \mathrm{~g} / \mathrm{L}$ sodium bicarbonate, $1 \%$ penicillin and streptomycin, 1.7 $\mathrm{mmol} / \mathrm{L}$ L-glutamine, non-essential amino acid, $55 \mathrm{mmol} / \mathrm{L}$ beta mercaptoethanol, $5 \mathrm{ng} / \mathrm{mL}$ mouse leukemia inhibitory factor (LIF), 3 $\mu \mathrm{mol} / \mathrm{L}$ EsK3 $\beta$ inhibitor, $1 \mu \mathrm{mol} / \mathrm{L}$ MEK inhibitor and $10 \% \mathrm{FBS}$. The $\mathrm{mESC}$ maintenance medium was used for spontaneous differentiation without supplementing LIF, the EsK3 $\beta$ inhibitor or the MEK inhibitor.

\section{Construction of the Clock knockout mESC line}

To construct the Clock knockout mESC line, we utilized the clustered, regularly interspaced, short palindromic repeats (CRISPR)/ CRISPR-associated protein 9 (CAS9) (CRISPR/CAS9) genomic 
editing tool. The CRISPR/CAS9 system is an efficient tool for genome engineering. It induces double-strand breaks (DSBs) and repair using the non-homologous end-joining (NHEJ) mechanism at any specific site. Briefly, one pair of independent oligo primers targeting exon 2 of the Clock gene was subcloned into the pX330 vector (Mizuno et al., 2014) (Addgene) to obtain the pX330-Clock plasmid. The primer sequences generating the guide RNA (gRNA) targeting the Clock gene were TCCATCTTTCTCGCGTT, then the complementary sequences were AGGTAGAAAGAGCGCAA. As the transcriptional promoter of $\mathrm{PX} 330$ plasmid was U6, we replaced base $T$ to base $\mathrm{G}$ for the first base was $\mathrm{G}$ to be effective transcription. With enzyme sites, the insertion sequences were as follows: oligo1: 5'CACCGCCATCTTTCTCGCGTTACC-3', oligo2: 5'-AAACGGTAACGCGAGAAAGAT GGC-3'. The pX330-Clock plasmid was transfected into single mESCs by electroporation. The correct knockout of Clock gene expression was confirmed by Western blot analysis and gene sequencing.

To consider the potential influence of off-target gene modifications (Yee, 2016), we detected the most probable ten off-target modification sites which were designed at the CRISPR website (http://crispr.mit.edu/), whose off-target cleavage site sequences and gene numbers were as follows in Table S3. Specific PCR primers were designed according to the target gene sequences of mouse (Table S3).

\section{Quantitative real-time PCR}

The mRNA expression levels of GAPDH, Oct4, Sox2, Klf4, Nanog, Zfp296, Eras, Dax1, Esg1, C-Myc, PCNA, CDK1, CDK2, CyclinD1, P27, Gata4, Sox17, Foxa2, Lamb1, BMP4, T, Eomes, Actc1, Nestin, Sox1, Neurod1, Otx1, Bcl-2, Bax, cleaved caspase-3, and caspase9 were quantified by RT-PCR. Specific PCR primers were designed according to the target gene sequences of mouse (Supplemental Table. 1). Cells were washed by ice-cold Phosphate buffer solution (PBS, $\mathrm{NaCl} 137 \mathrm{mmol} / \mathrm{L}, \mathrm{KCl} 2 \mathrm{mmol} / \mathrm{L}, \mathrm{Na}_{2} \mathrm{HPO}_{4} 10 \mathrm{mmol} / \mathrm{L}$,

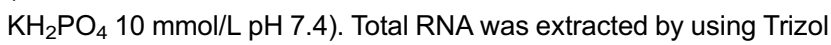
reagent (Invitrogen, USA). Qualities of extracted RNA qualities were measured by $\mathrm{OD}_{260} / \mathrm{OD}_{280}$ ratios which ranged from 1.9 to 2.1 . The cDNA was obtained through reverse transcription kit according to manufacturer's instructions (TOYOBO, Japan). The amplification mixture comprised $1 \mu \mathrm{L}$ of RT reaction mix, $10 \mu \mathrm{L}$ of SYBR $®$ Premix Ex Taq TM $(2 \times)$ (TaKaRa, China), $0.5 \mu \mathrm{L}$ of $10 \mu \mathrm{mol} / \mathrm{L}$ each of primers and $8.5 \mu \mathrm{L} \mathrm{ddH}_{2} \mathrm{O}$. Reactions were performed on a fluorescence temperature cycler (Bio-Rad, Hercules, CA, USA). The PCR conditions for Clock and GAPDH were as follows: one cycle of $3 \mathrm{~min}$ at $95^{\circ} \mathrm{C} ; 35$ cycles of $15 \mathrm{~s}$ at $95^{\circ} \mathrm{C}, 30 \mathrm{~s}$ at $58^{\circ} \mathrm{C}, 30 \mathrm{~s}$ at $72^{\circ} \mathrm{C}$. The primer sequences are listed in Supplemental Table 1. The threshold cycle (CT) in RT-PCR was analyzed using the $2^{-\Delta \Delta C t}$ method

\section{Western blot}

Total protein was extracted from the treated cells using Radio-Immunoprecipitation Assay (RIPA) lysis buffer $(50 \mathrm{mmol} / \mathrm{L} \mathrm{Tris} / \mathrm{HCl} \mathrm{pH}$ 7.4, $150 \mathrm{mmol} / \mathrm{L} \mathrm{NaCl}, 1 \%$ Nonidet-P40, 0.5\% Sodium deoxycholate, $0.1 \%$ SDS). The extraction and isolation of nuclear protein were performed according to the instructions in the Nuclear and Cytoplasmic Protein Extraction Kit (Beyotime, China). Equal amounts $(30-50 \mu \mathrm{g})$ of protein extracted from cells were boiled at $100^{\circ} \mathrm{C}$ in $1 \times$ SDS loading buffer for $10 \mathrm{~min}$ and then were loaded and separated by sodium dodecyl-sulfate (SDS)-polyacrylamide gel electrophoresis (PAGE). Proteins were transferred to a polyvinylidene fluoride (PVDF) membrane (Millipore, Billerica, MA, USA), blocked by $5 \%$ nonfat dry milk in Tris-buffered saline containing Tween-20. The membranes were incubated with antibodies against PKM2 (Cell Signaling), CK19 (Abcam), and EpCAM (Abcam). Signals were detected with horseradish peroxidase (HRP)-conjugated goat anti-mouse or goat anti-rabbit IgG. Immunoreactive bands were visualized by enhanced chemiluminescence (ECL, Amersham Biosciences), which was developed and quantified using an imaging system (Tanon, China).

\section{Alkaline phosphatase staining}

The sections were washed with cold $1 \times$ PBS three times, treated with $4 \%$ paraform- aldehyde for 1 to $2 \mathrm{~min}$, washed with cold $1 \times$ PBS twice, and with $1 \times$ Tris buffer solution tween (TBST, $50 \mathrm{mmol} / \mathrm{L}$ Tris, $150 \mathrm{mmol} / \mathrm{L} \mathrm{NaCl}, 1 \%$ Tween-20) once, followed by staining with Alkaline Phosphatase Kit (Millipore, USA) according to the manufacturer's instructions. Images were taken by a Leica DMi8 microscope (Leica, Germany) and analyzed using Image Pro Plus 6.0 software (Media Cybernetics, Rockville, MD).

\section{Teratoma formation assay}

To study the pluripotency of wild type mESCs and Clock knockout mESCs in vivo, we performed teratoma formation assay which could conduct as a tool for monitoring pluripotency in stem cell research (Nelakanti et al., 2015). We injected $1 \times 10^{7}$ wild type mESCs and Clock knockout mESCs into the oxters of immunodeficiency mice respectively. 1-2 cm teratoma formed after four weeks. Six weeks later, we detected the differential capacity of wild type mESCs and Clock knockout mESCs by hematoxylin-eosin staining (HE staining). We found that both wild type mESCs and Clock knockout mESCs could differentiate to endoderm, mesoderm, and ectoderm layers cells, which indicated that they have similar potential of multilineage differentiation in vivo.

\section{Immunofluorescent staining}

The sections were washed with cold $1 \times$ PBS three times, and treated with $4 \%$ paraformaldehyde for $15 \mathrm{~min}$. They were again washed with cold $1 \times$ PBS three times, and treated with $5 \%$ Triton X100 in Tris buffer solution (TBS, $50 \mathrm{mmol} / \mathrm{L}$ Tris/HCl pH 7.4, 150 $\mathrm{mmol} / \mathrm{L} \mathrm{NaCl}$ ) for $20 \mathrm{~min}$ at room temperature (RT) (membrane proteins do not need this step). After another wash with cold $1 \times$ PBS three times for $5 \mathrm{~min}$, they were treated with normal goat serum for $30 \mathrm{~min}$ at $\mathrm{RT}$, followed by the addition of primary antibodies (1:200) and incubation in a wet box for $2 \mathrm{~h}$ at RT or $4{ }^{\circ} \mathrm{C}$ overnight. Fluorescent secondary antibodies (1:350) were added and the cells were incubated for $30 \mathrm{~min}$ or $1 \mathrm{~h}$ at RT, washed three times for 5 min each with PBST (1\% Tween in PBS buffer). It was followed by staining with 2-(4-Amidinophenyl)-6-indolecarbamidine dihydrochloride (DAPI) (1:1000) for $5 \mathrm{~min}$ at RT, and washed three times for 5 min each with PBS. After treatment with a fluorescence quenching agent, the specimens were sealed and photographed under fluorescence and laser confocal microscope. 
The images were analyzed using Image Pro Plus 6.0 software (Media Cybernetics, Rockville, MD).

\section{Cell proliferation curve}

Cell viability and number was detected using a cell counting chamber. Wild type mESCs and Clock knockout mESCs were transferred to 12-well and 6-well plates (Corning Inc., Corning, New York, USA) at $1 \times 10^{4}$ cells/well and cultured at 6 different time points $(24,48,72,96,120$ and $144 \mathrm{~h})$. At the indicated time, cells were digested with $0.05 \%$ trypsin, and $20 \mu \mathrm{L}$ of the resuspended cells were collected in the cell counting chamber (Nexcelom) and counted using the Cellometer Mini software.

Induction of biological rhythms with horse serum shock

Briefly, we induced the biological rhythms of wild type mESCs and Clock knockout mESCs after spontaneous differentiation with horse serum shock as previously described (Balsalobre et al., 1998; Xiang et al., 2012). The wild type mESCs and Clock knockout mESCs were cultured in the mESC maintenance medium and differentiated spontaneously for 15 days in the differentiation medium. The medium was removed and cells were washed with $1 \times$ PBS, followed by addition of the differentiation medium without FBS for $24 \mathrm{~h}$. The medium was removed and cells were washed with $1 \times$ PBS. A new differentiation medium containing $50 \%$ horse serum was added to the cells. After incubation for $2 \mathrm{~h}$, the medium was removed and washed with $1 \times \mathrm{PBS}$, and replaced with the original differentiation medium.

\section{Annexin V and PI assay}

The FITC Annexin V and Propidium lodide (PI) Apoptosis Detection Kit II (Pharmingen, USA) were used to analyze apoptosis and cell death rate using flow cytometry. The wild type mESCs and Clock knockout mESCs were collected and washed twice with cold PBS. The resuspended cells in $100 \mu \mathrm{L}$ of $1 \times$ binding buffer were transferred to a $1.5 \mathrm{~mL}$ culture tube, and $5 \mu \mathrm{L}$ of FITC Annexin $\mathrm{V}$ and PI were added to each tube. After gentle vortexing, the cells were incubated for $15 \mathrm{~min}$ at room temperature in the dark. Finally, $400 \mu \mathrm{L}$ of the $1 \times$ binding buffer was added to each tube, and analyzed by flow cytometry in $1 \mathrm{~h}$.

\section{Statistical analysis}

All the data were expressed as means $\pm S D$. The statistical significance of differences between the experimental groups was determined using one-way ANOVA by Student's t-test (SPSS 11.0, SPSS Inc., Cary, NC). Probability values $(p)$ of less than 0.05 were considered statistically significant.

\section{ACKNOWLEDGEMENTS}

This work was supported by the National Science Foundation Fostering Talents in Basic Research of China No. J1210041 (RZ.Q.); the National Natural Science Foundation of China [No. 81322003 (N.S.), No.31571527 (N.S.), No. 81570771 (RZ.Q.)]; Recruitment Program of Global Experts of the Organization Department of the Central Committee of the CPC (N.S.); Science and Technology Commission of Shanghai Municipality (No. 13JC1401704) (N.S.); and Shanghai
Key Laboratory of Clinical Geriatric Medicine No. $13 \mathrm{dz} 2260$ 700 (C.L).

\section{COMPLIANCE WITH ETHICS GUIDELINES}

Chao Lu, Yang Yang, Ran Zhao, Bingxuan Hua, Chen Xu, Zuoqin Yan, Ning Sun and Ruizhe Qian declare that they have no conflict of interest.

All institutional and national guidelines for the care and use of laboratory animals were followed.

\section{OPEN ACCESS}

This article is distributed under the terms of the Creative Commons Attribution 4.0 International License (http://creativecommons.org/ licenses/by/4.0/), which permits unrestricted use, distribution, and reproduction in any medium, provided you give appropriate credit to the original author(s) and the source, provide a link to the Creative Commons license, and indicate if changes were made.

\section{REFERENCES}

Balsalobre A, Damiola F, Schibler U (1998) A serum shock induces circadian gene expression in mammalian tissue culture cells. Cell 93:929-937

Borgs L, Beukelaers P, Vandenbosch R, Nguyen L, Moonen G, Maquet P, Albrecht U, Belachew S, Malgrange B (2009) Period 2 regulates neural stem/progenitor cell proliferation in the adult hippocampus. BMC Neurosci 10:30

Cardinal-Aucoin M, Steel CG (2016) Circadian control of prothoracicotropic hormone release in an adult insect and the induction of its rhythmicity by light cues. Comp Biochem Physiol A Mol Integr Physiol 201:46-52

Casanova-Acebes M, Pitaval C, Weiss LA, Nombela-Arrieta C, Chevre R, Noelia AG, Kunisaki $Y$, Zhang D, van Rooijen N, Silberstein LE et al (2013) Rhythmic modulation of the hematopoietic niche through neutrophil clearance. Cell 153:1025-1035

Dashti HS, Aslibekyan S, Scheer FA, Smith CE, Lamon-Fava S, Jacques P, Lai CQ, Tucker KL, Arnett DK, Ordovas JM (2016) Clock genes explain a large proportion of phenotypic variance in systolic blood pressure and this control is not modified by environmental temperature. Am J Hypertens 29:132-140

Debruyne JP (2008) Oscillating perceptions: the ups and downs of the CLOCK protein in the mouse circadian system. J Genet 87:437-446

DeBruyne JP, Weaver DR, Reppert SM (2007a) CLOCK and NPAS2 have overlapping roles in the suprachiasmatic circadian clock. Nat Neurosci 10:543-545

DeBruyne JP, Weaver DR, Reppert SM (2007b) Peripheral circadian oscillators require CLOCK. Curr Biol 17:R538-539

Dubrovsky YV, Samsa WE, Kondratov RV (2010) Deficiency of circadian protein CLOCK reduces lifespan and increases agerelated cataract development in mice. Aging (Albany NY) 2:936-944

Dunlap JC (1999) Molecular bases for circadian clocks. Cell 96:271290 
Fu L, Pelicano H, Liu J, Huang P, Lee C (2002) The circadian gene Period2 plays an important role in tumor suppression and DNA damage response in vivo. Cell 111:41-50

Gachon F, Nagoshi E, Brown SA, Ripperger J, Schibler U (2004) The mammalian circadian timing system: from gene expression to physiology. Chromosoma 113:103-112

Gaddameedhi S, Selby CP, Kemp MG, Ye R, Sancar A (2015) The circadian clock controls sunburn apoptosis and erythema in mouse skin. J Invest Dermatol 135:1119-1127

Hu K, Van Someren EJ, Shea SA, Scheer FA (2009) Reduction of scale invariance of activity fluctuations with aging and Alzheimer's disease: Involvement of the circadian pacemaker. Proc Natl Acad Sci USA 106:2490-2494

Janich P, Toufighi K, Solanas G, Luis NM, Minkwitz S, Serrano L, Lehner B, Benitah SA (2013) Human epidermal stem cell function is regulated by circadian oscillations. Cell Stem Cell 13:745-753

Johnson MH, Lim A, Fernando D, Day ML (2002) Circadian clockwork genes are expressed in the reproductive tract and conceptus of the early pregnant mouse. Reprod Biomed Online 4:140-145

Johnston JD, Ordovas JM (2016) Circadian rhythms, metabolism, and chrononutrition in rodents and humans. Adv Nutr 7:399-406

Karpowicz P, Zhang Y, Hogenesch JB, Emery P, Perrimon N (2013) The circadian clock gates the intestinal stem cell regenerative state. Cell Rep 3:996-1004

King DP, Zhao Y, Sangoram AM, Wilsbacher LD, Tanaka M, Antoch MP, Steeves TD, Vitaterna MH, Kornhauser JM, Lowrey PL et al (1997) Positional cloning of the mouse circadian clock gene. Cell 89:641-653

Koike N, Yoo SH, Huang HC, Kumar V, Lee C, Kim TK, Takahashi JS (2012) Transcriptional architecture and chromatin landscape of the core circadian clock in mammals. Science 338:349-354

Kovanen L, Saarikoski ST, Aromaa A, Lonnqvist J, Partonen T (2010) ARNTL (BMAL1) and NPAS2 gene variants contribute to fertility and seasonality. PLoS One 5:e10007

Li R, Cheng S, Wang Z (2015) Circadian clock gene plays a key role on ovarian cycle and spontaneous abortion. Cell Physiol Biochem 37:911-920

Liang X, Cheng S, Jiang X, He X, Wang Y, Jiang Z, Hou W, Li S, Liu Y, Wang $Z$ (2013) The noncircadian function of the circadian Clock gene in the regulation of male fertility. J Biol Rhythms 28:208-217

Lopez-Minguez J, Gomez-Abellan P, Garaulet M (2016) Circadian rhythms, food timing and obesity. Proc Nutr Soc. doi:10.1017/ S0029665116000628

Lowrey PL, Takahashi JS (2004) Mammalian circadian biology: elucidating genome-wide levels of temporal organization. Annu Rev Genomics Hum Genet 5:407-441

McAlpine CS, Swirski FK (2016) Circadian influence on metabolism and inflammation in atherosclerosis. Circ Res 119:131-141

Mizuno S, Dinh TT, Kato K, Mizuno-lijima S, Tanimoto Y, Daitoku Y, Hoshino Y, Ikawa M, Takahashi S, Sugiyama F et al (2014) Simple generation of albino C57BL/6J mice with G291T mutation in the tyrosinase gene by the CRISPR/Cas9 system. Mamm Genome 25:327-334

Mukherjee S, Coque L, Cao JL, Kumar J, Chakravarty S, Asaithamby A, Graham A, Gordon E, Enwright JF III, DiLeone RJ et al (2010) Knockdown of Clock in the ventral tegmental area through RNA interference results in a mixed state of mania and depression-like behavior. Biol Psychiatry 68:503-511

Nelakanti RV, Kooreman NG, Wu JC (2015) Teratoma formation: a tool for monitoring pluripotency in stem cell research. Curr Protoc Stem Cell Biol 32(4a.8):1-17

Nitschke S, Cortleven A, Iven T, Feussner I, Havaux M, Riefler M, Schmulling T (2016) Circadian stress regimes affect the circadian clock and cause jasmonic acid-dependent cell death in cytokinindeficient arabidopsis plants. Plant Cell. doi:10.1105/tpc.16.00016

Peyric E, Moore HA, Whitmore D (2013) Circadian clock regulation of the cell cycle in the zebrafish intestine. PLoS One 8:e73209

Tobback J, Vuerinckx K, Boerjan B, Huybrechts R (2012) RNA interference mortality points to noncircadian functions for the clock gene in the desert locust Schistocerca gregaria. Insect Mol Biol 21:369-381

Tracey CJ, Pan X, Catterson JH, Harmar AJ, Hussain MM, Hartley PS (2012) Diurnal expression of the thrombopoietin gene is regulated by CLOCK. J Thromb Haemost 10:662-669

Witting W, Kwa IH, Eikelenboom P, Mirmiran M, Swaab DF (1990) Alterations in the circadian rest-activity rhythm in aging and Alzheimer's disease. Biol Psychiatry 27:563-572

Wood PA, Yang X, Taber A, Oh EY, Ansell C, Ayers SE, Al-Assaad Z, Carnevale K, Berger FG, Pena MM et al (2008) Period 2 mutation accelerates ApcMin/+ tumorigenesis. Mol Cancer Res 6:1786-1793

Wyse CA, Coogan AN (2010) Impact of aging on diurnal expression patterns of CLOCK and BMAL1 in the mouse brain. Brain Res 1337:21-31

Xiang S, Mao L, Duplessis T, Yuan L, Dauchy R, Dauchy E, Blask DE, Frasch T, Hill SM (2012) Oscillation of clock and clock controlled genes induced by serum shock in human breast epithelial and breast cancer cells: regulation by melatonin. Breast Cancer (Auckl) 6:137-150

Xue T, Song C, Wang Q, Wang Y, Chen G (2016) Investigations of the CLOCK and BMAL1 proteins binding to DNA: a molecular dynamics simulation study. PLoS One 11:e0155105

Yagita K, Horie K, Koinuma S, Nakamura W, Yamanaka I, Urasaki A, Shigeyoshi Y, Kawakami K, Shimada S, Takeda J et al (2010) Development of the circadian oscillator during differentiation of mouse embryonic stem cells in vitro. Proc Natl Acad Sci USA 107:3846-3851

Yang G, Chen L, Grant GR, Paschos G, Song WL, Musiek ES, Lee V, McLoughlin SC, Grosser T, Cotsarelis G et al (2016) Timing of expression of the core clock gene Bmal1 influences its effects on aging and survival. Sci Transl Med 8:324316

Yee JK (2016) Off-target effects of engineered nucleases. FEBS J. doi:10.1111/febs. 13760 